\title{
Algebraic Learning and Neural Network Models for Transitive and Non-transitive Responding
}

\author{
Martina Siemann and Juan D. Delius \\ Allgemeine Psychologie, Universität Konstanz, Konstanz, Germany
}

\begin{abstract}
Transitive inference is a kind of deductive reasoning. Given the premises "Anna is taller than Paul" and "Paul is taller than Mary", adults and older children easily conclude that "Anna is taller than Mary". However, a related transitive responding ability has also been demonstrated in younger children and some animals with non-verbal tasks. For this, the premise statements are converted into an operant discrimination task. The subjects are offered the stimulus pairs $\mathrm{A}+\mathrm{B}-, \mathrm{B}+\mathrm{C}-, \mathrm{C}+\mathrm{D}-, \mathrm{D}+\mathrm{E}-$ where + signifies that the choice of the relevant stimulus is rewarded and - indicates that its choice is penalised. When the subjects responded correctly to these premises, unreinforced tests with the conclusion stimulus pair BD were conducted. If they preferentially chose B, they were said to respond transitively. Algebraic conditioning models have been shown to be capable of reproducing such transitive behaviour. We describe a particularly simple algebraic model based on instrumental conditioning and then develop a neural network that yields transitive responding based on similar principles as the model. A variant of the model also incorporates a value transfer mechanism based on a classical conditioning process that appears to contribute occasionally to the itemordering underlying transitivity. Some humans, however, exhibit good premise pair performance but poor conclusion test performance. We consider model and network modifications that can account for this behaviour. A variant called the $\varepsilon \kappa$ model is shown to yield graded degrees of transitive responding with conclusion pairs while maintaining good performance on premise pairs.
\end{abstract}

Requests for reprints should be addressed to Martina Siemann, Algemeine Psychologie, Universität Konstanz, D-78434 Konstanz, Germany. E-mail: martina.siemamn(eunikonstanz.de

This research was supported by the Deutsche Forschungsgemeinschaft. We thank Professor J. Emmerton of Purdue University for comments on a previous draft of the manuscript, Ms A. McLintock for help with preparation of the manuscript and Ms C. Roberts for improving the English.

Konstanzer Online-Publikations-System (KOPS)

URN: http://nbn-resolving.de/urn:nbn:de:bsz:352-205959 


\section{INTRODUCTION}

In recent years, there has been an increasing interest in the non-verbal inferential competencies of humans and, indeed, animals. The starting point was classical verbal transitivity syllogisms, where presentations of premises of the type "Anna is taller than Paul" and "Paul is taller than Mary" were expected to lead to the conclusion "Anna is taller than Mary". Piaget (1921; cf. Breslow, 1981) tested children of different ages with this type of task and found that only children older than about 7 years could conclude correctly in a reliable manner. However, when Bryant and Trabasso (1971) converted the problem into a largely nonverbal task, where the premises involved the presentation of pairs of five differently coloured sticks of differing length according to the scheme $\mathrm{A}>\mathrm{B}, \mathrm{B}>\mathrm{C}, \mathrm{C}>\mathrm{D}, \mathrm{D}>\mathrm{E}$, they found that children aged only 4 years behaved transitively by preferring $B$ when presented with the conclusion pair B ? D, provided that they had previously memorised the premise pairs. The reasons for expanding the task to five, rather than three, items will become obvious later. McGonigle and Chalmers (1977), who were concerned with the inferential capacities of squirrel monkeys, went on to convert the task into a fully non-verbal, multiple-operant discrimination. In it, the premises are first conveyed to the subjects by repeatedly presenting stimulus pairs in an essentially random order, according to the scheme $\mathrm{A}+\mathrm{B}-, \mathrm{B}+\mathrm{C}-, \mathrm{C}+\mathrm{D}-$ and $\mathrm{D}+\mathrm{E}-$, where + indicates that the choice of the relevant stimulus was rewarded and - signifies that its choice was penalised, which often involved only non-reward. When subjects have learned to deal correctly with these inequalities, they are faced with conclusion tests involving the presentation of stimulus pairs $\mathrm{BD}$ in unreinforced trials. Transitive responding is said to occur when stimulus $B$ is preferred to $D$. Note that during premise training both $B$ and $D$ are in principle scheduled to be equally often rewarded and penalised.

Based on variations of this general method, it has been shown that, in addition to squirrel monkeys (McGonigle \& Chalmers, 1992), macaques (Treichler \& Van Tilburg, 1996), chimpanzees (Boysen, Berntson, Shreyer, \& Quigley, 1993; Gillan, 1981), rats (Davis, 1992a; Roberts \& Phelps, 1994) and pigeons (Fersen, Wynne, Delius, \& Staddon, 1991; Siemann, 1993b; Siemann, Delius, \& Wright, 1996; Wynne, 1997), children (Chalmers \& McGonigle, 1984) and adult humans (Siemann, 1993a,b, 1994a,b; Siemann \& Delius, 1993, 1996; Werner, Koeppl, \& Delius, 1992) do indeed respond transitively. Although still a contentious issue (see, for example, Markovits \& Dumas, 1992) most of the aforementioned investigators have assumed that the information processing underlying the solution of non-verbal 
syllogisms and verbal syllogisms involves at least some commonalities. We return to this issue later.

Traditional explanations of transitive inference appealed to the rules of formal logic, sometimes by referring to a so-called mental logic-that is, to the involvement of rational processes that obey the rules of logic. Humans were thought to draw deductions through a coordination of the various premises by explicitly applying logical schemes based on language codes that develop during childhood (Piaget, 1953). Recently, authors have begun to doubt the validity of this kind of account. Everyday reasoning demonstrably accords quite poorly with the rules of formal logic and there has been an increasing tendency to appeal to a so-called mental paralogic based on imagery-like operations (DeSoto, London, \& Handel, 1965; Johnson-Laird, 1986). Also, behaviour that conforms with such reasoning often seems to occur dissociated from the awareness that seems necessary for proper rational thought. Some transitively successful subjects cannot report verbally the crucial structure of the task and the logical procedure that mediates its solution. That task structure awareness and actual task performance are not necessarily correlated was demonstrated in a recent series of transitive responding experiments with adult humans. The concreteness of the premise stimuli and the relations between them were varied across experiments (Siemann, 1993a, 1996; Siemann \& Delius, 1993, 1996). For example, one experiment involved the discrimination of pairs of random polygons presented in random order within a computer game that related six of these stimuli through reinforcement allocations in the style of the non-verbal task sketched above (Fig. 1). Another more naturalistic experiment used named human figures additionally related through visible size differences and was effectively quite close in format to the verbal syllogisms alluded to earlier. Although the proportion of subjects verbalising awareness about the transitive structure of the problems increased with the concreteness of the task, this did not influence significantly the objective performance during the conclusion tests, as measured by choice accuracies.

As already stated, accounts of deductive reasoning based on a mental imagery metaphor have gained favour in the same measure as formal logic theory has lost its attraction (Johnson-Laird, 1986). The metaphor assumes that subjects construct an orderly spatial representation of the items based on the images evoked by the premises. In the case of the verbal transitive syllogism cited earlier, the premises would, for example, induce the construction of a kind of line-up according to size (mental line; Evans, Newstead, \& Byrne, 1993; Trabasso \& Riley, 1975) of personalised images of Anna, Paul and Mary in visual memory, the build-up of this representation probably beginning with the end-items Anna and Mary. To arrive at the correct answer to the concluding question, 


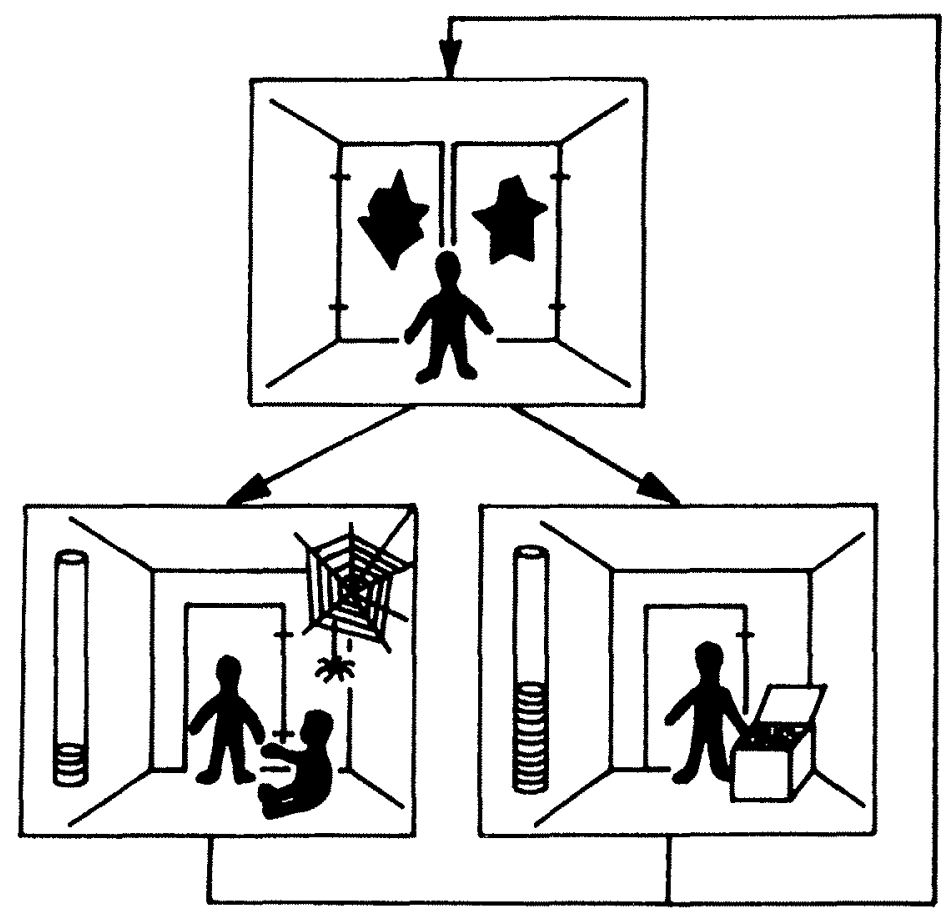

FIG. 1. A computer game used for transitive responding experiments in humans. The subject steers a figurine through an imaginary castle using the computer keyboard. The figurine repeatedly encounters pairs of doors displaying pairs of polygons, an example of which is shown. If the subject chooses the door bearing the polygon defined as correct, the figurine enters a treasure chamber and collects a symbolic coin from a chest. If the subject chooses the incorrect door, the figurine enters a chamber of horrors and loses a coin to a beggar. The allocation of correct and incorrect polygons to left and right doors was done quasi-randomly from trial to trial (modified from Siemann, 1994a).

subjects would then only need to scan this representation with their hypothetical mind's eye. Aware subjects in fact frequently report using such a strategy, sometimes even when faced with an abstract non-verbal syllogism such as the differentially reinforced polygon task mentioned above (Siemann, 1993a). This type of explanation, when elaborated in more detail, is also in reasonable qualitative agreement with some special effects often observed in transitive inference studies (Breslow, 1981). The theory, however, has understandably not yet been stated in a computable form. Thus, so far, no stringent quantitative extrapolations can easily be derived from it.

Contrasting with that, Harris and McGonigle (1994) have proposed an at least partially computable artificial intelligence model that is capable of 
transitive responding. It relies on propositional operations that can be viewed as related to the application of logic rules. It involves a number of decision prescriptions that have to be dealt with in strict order when facing a stimulus pair. For a given transitivity task, there are a number of different stacks of rules that will lead to correct transitive conclusions. The particular stack used by a given individual is thought to be learned through a trial-and-error process when the subject deals with the premise pairs. An example of a correct stack for the above $A+$ to $E-$ transitivity task is: A present, choose A; E present, avoid E; D present, avoid D; B present, avoid $B$, where the third rule is the one that will solve the $B D$ conclusion pair. Different rule-stacks can realistically mimic individual performance details when transitivity tasks of conventional design are involved. However, with non-standard designs such as those involving two independent, or quasi-independent, item series (Siemann \& Delius, 1996; Werner et al., 1992; see later), the stack modelling becomes quite unwieldy. Also, propositional models are difficult to convert into neural networks, an issue that particularly concerns us here.

\section{LUCE'S MODEL}

Models of transitive responding that are, in our view, preferable from both the plausibility and the computability points of view are those based on learning theory principles. All those models that have been proposed to account for transitive responding are, moreover, in some sense compatible with the mental line model in so far as all of them rely on the buildup of a kind of graded representation of the stimuli. A first model of this kind (value transfer theory) was proposed in connection with the demonstration that pigeons are capable of responding transitively (Fersen, Wynne, Delius, \& Staddon, 1990, 1991). This model was criticised by Couvillon and Bitterman (1992) for purportedly making unrealistic assumptions. They proceeded to propose another, in their opinion, more straightforward model based on Bush and Mosteller's (1951) learning operator. Other such learning models of transitive responding were formulated soon afterwards (Davies, Staddon, Machado, \& Palmer, 1993; Wynne, Fersen, \& Staddon, 1992; see also Siemann, 1993a, 1994a; Wynne, 1995, 1997). Among these models, all of which emulate transitive responding reasonably well, we found that one involving little algebra and few parameters accounted quite satisfactorily for several transitive responding results (Siemann, 1993a; Siemann \& Delius, 1993; Werner et al., 1992). It is based on Luce's (1959) beta learning operator. Here we enlarge on this particular model because, as we show later, it lends itself for conversion into a minimal and intelligible neural network equivalent. 
The algebraic version of Luce's model assumes that the associative (response eliciting) value of a stimulus is increased by a certain amount if the choice of that stimulus is followed by a reward, and decreased by a certain amount if it is followed by a penalty. Thus, given that the stimulus pair $\mathrm{X}+\mathrm{Y}$ - (standing in for training pairs such as those listed earlier) is presented on a certain trial, and given that the subject chooses $X+$, and is thus rewarded, this stimulus value is updated according to $\mathrm{V}_{\mathrm{x}} \leftarrow \mathrm{V}_{\mathrm{x}}+\left(\mathrm{V}_{\mathrm{x}} \times \beta_{+}\right)$, where $\beta_{+}$is a learning parameter corresponding to reward. The symbol $\leftarrow$ indicates that, after a choice, the left-hand variable assumes the magnitude of the right-hand expression. This expression specifies the new magnitude on the basis of the previous magnitude of that same variable. The value of $Y$ in this instance remains unchanged according to $V_{y} \leftarrow V_{y}$. If, however, the subject chooses $Y$ and is accordingly penalised, then the value of that stimulus is updated according to $\mathrm{V}_{\mathrm{y}} \leftarrow \mathrm{V}_{\mathrm{y}}-\left(\mathrm{V}_{\mathrm{y}} \times \beta_{\mathrm{L}}\right.$ ), where $\beta$ is a parameter corresponding to penalty. In this instance, it is the value of $X$ that remains unchanged according to $V_{x} \leftarrow V_{x}$ (Wynne et al., 1992). The current values $V_{x}$ and $V_{y}$ in turn determine the probability with which $X$ will be chosen in preference to $Y$ in a given trial according to $\mathrm{p}_{\mathrm{x} y}=\mathrm{V}_{\mathrm{x}} /\left(\mathrm{V}_{\mathrm{x}}+\mathrm{V}_{\mathrm{y}}\right)$.

These equations are sufficient to simulate the transitive task learning by individual subjects. But it is practical to expand the model so that it applies to a population of subjects. Consider a hypothetical infinite number of identical subjects involved in precisely the same trial. There will obviously be a fraction $p_{x y}$ of subjects choosing stimulus $X$ and a remaining fraction $1-p_{x y}$ of subjects choosing stimulus $Y$. The population-wide average updates after completion of a trial $X+Y$ - will thus be $V_{x} \leftarrow V_{x}+\left(p_{x y} \times V_{x} \times \beta_{+}\right)$and $V_{y} \leftarrow V_{y}-\left(1-p_{x y}\right) \times V_{y} \times \beta \ldots$. If the model is correct, the actual mean updates of a finite, real group of subjects can be assumed to approximate these theoretical value updates. Note that this model relies on a very simple choice mechanism and that its stimulus values $V_{s}$ are not bounded. The transitive responding results so far available do not really warrant the introduction of any upper bounds for the stimulus values (Siemann et al., 1996; but see later).

Simulations with the Luce model, as specified above, yield transitive responding outcomes for the critical, unreinforced $\mathrm{BD}$ tests that reflect an underlying $V_{b}>V_{d}$ inequality. Given the same (or very similar) initial values for $V_{a}, V_{b}, V_{c}, V_{d}, V_{c}$, and any values for $\beta_{+}, \beta$ which yield a successful learning of the stimulus pairs $A+B-B+C-, C+D-$ and $\mathrm{D}+\mathrm{E}-$ repeatedly presented in random order, these formulae do indeed result ultimately in stimulus values obeying the inequalities $V_{a}>V_{b}>V_{c}>V_{d}>V_{c}$. This value ranking, somewhat surprisingly, emerges despite the circumstance that the stimuli seem scheduled to yield a $V_{a}>V_{b}=V_{c}=V_{c}>V_{c}$ ranking. The fact is, contrary to the intention 
of the training design, the middle stimuli $B, C$ and $D$ turn out not to be equally often rewarded and penalised. A choice/reinforcement biasing/ balancing process that arises during training interferes with the design's intent (Couvillon \& Bitterman, 1992; Siemann \& Delius, 1996; Siemann et al., 1996). This process occurs because the changes in stimulus values caused by the differential reinforcements modify the stimulus choice probabilities, and vice versa, in an interactive manner. To understand how the value inequalities come about, we consider the training effects associated with, firstly, the pairs $\mathrm{A}+\mathrm{B}-, \mathrm{B}+\mathrm{C}-$ and, secondly, the pairs $\mathrm{E}-\mathrm{D}+, \mathrm{D}-\mathrm{C}+$. Because choices of stimulus $\mathrm{A}$ are scheduled to be always rewarded, the value $V_{a}$ increases steeply come what may. The probability $p_{b a}$ of $B$ being chosen and penalised within pair $A+B$-consequently decreases rapidly. The value $V_{b}$ of $B$ thus decreases very little within this pair. This automatically ensures that choices of $B$ within the pair $\mathrm{B}+\mathrm{C}-$ are basically quite probable. As rewarded choices of $\mathrm{B}$ within this pair accumulate, both $V_{b}$ and $p_{b c}$ increase steadily. Of course, $V_{b}$ cannot increase as much as $V_{a}$ because occasional penalised choices of $B$ within pair $A+B$ - ensure the inequality $V_{a}>V_{b}$ is maintained. Overall, however, choices of $B$ are more often rewarded than penalised and $V_{b}$ thus attains a comparatively large magnitude.

As any choices of $E$ are scheduled to be always penalised, $V_{c}$ inevitably decreases. Because of that, but also because choices of $D$ are rewarded, both $p_{d c}$ and $V_{d}$ tend to increase within pair $E-D+$. This automatically ensures that choices of $\mathrm{D}$ within the pair $\mathrm{D}-\mathrm{C}+$ remain rather probable. The choices of $D$ within this latter pair are penalised and cause $V_{a}$ and $p_{d c}$ to fall again. Choices of $D$ are thus overall similarly often rewarded and penalised, meaning that $V_{d}$ only attains a moderate magnitude, markedly less than that corresponding to $V_{b}$ but of course more than $V_{c}$, which belongs to the always penalised $E$. The value $V_{c}$ of the bridging stimulus $C$ necessarily acquires an intermediate magnitude between $V_{b}$ and $V_{d}$ within this process, thus completing the $V_{a}>V_{b}>V_{c}>V_{c l}>V_{c}$ ranking alluded to above. Note that the value ranking brought about by Luce's model (and, indeed, by other related learning models) bears some similarity to the idea that humans solve transitive inference tasks by building up, and then recurring to, a linearly ordered imaginal representation of the premise items, discussed in the previous section.

With appropriately chosen $\beta_{+}$and $\beta$ parameters and the same initial values for all stimuli (the precise value magnitude is unimportant but conveniently small), the Luce model yields approximations to the results of varied non-verbal transitivity experiments, even when these involve more than five stimulus items, forecasting a transitive performance on unreinforced test pairs composed of non-adjacent stimuli, for example for 
TABLE 1

Comparison of Percent Mean Accuracies Scored by Human Subjects $(n=15)$ who Achieved Transitivity in a Non-verbal Transitivity Experiment, and Expected Percentages According to a Simulation with Luce's Model (from Siemann \& Delius, 1993)

\begin{tabular}{|c|c|c|c|c|c|c|c|c|}
\hline & \multicolumn{5}{|c|}{ Premise Pains } & \multicolumn{3}{|c|}{ Conclusion Pairs } \\
\hline & $A B$ & $B C$ & $C D$ & $D E$ & $E F$ & $B E$ & $B D$ & $C E$ \\
\hline Expected & 98 & 96 & 95 & 92 & 99 & 100 & 100 & 100 \\
\hline Observed & 98 & 97 & 95 & 96 & 99 & 96 & 97 & 87 \\
\hline
\end{tabular}

$\mathrm{XZ}$ according to $\mathrm{p}_{\mathrm{x} z}=\mathrm{V}_{\mathrm{x}} /\left(\mathrm{V}_{\mathrm{x}}+\mathrm{V}_{\mathrm{z}}\right)$, whenever a more than $50 \%$ correct performance on the premise pairs is obtained after sufficient training. As an example, Table 1 compares the results of a simulation with Luce's model with the mean accuracy of transitivity achieved by human subjects on the various training and test pairs in the final phase of the random polygon experiment mentioned earlier (Siemann \& Delius, 1993; for further simulations, see Delius \& Siemann, 1998; Siemann, 1993a, 1994a; Werner et al., 1992).

In a somewhat more involved experiment (Siemann, 1994a), subjects were trained with the overlapping pairs of two parallel four-term series that shared the same end stimuli but differed regarding the middle stimuli $\left(\mathrm{A}+\mathrm{B}_{1^{-}}, \quad \mathrm{B}_{1}+\mathrm{C}_{1-}, \quad \mathrm{C}_{1}+\mathrm{D}-; \mathrm{A}+\mathrm{B}_{2-}, \mathrm{B}_{2}+\mathrm{C}_{2-}, \mathrm{C}_{2}+\mathrm{D}-\right)$. After the subjects had learned all these pairs, they were tested in unreinforced trials with the middle stimuli paired across the series $\left(B_{1} C_{2}, B_{2} C_{1}, B_{1} B_{2}, C_{1} C_{2}\right)$. The subjects that responded transitively chose preferentially stimuli $B_{1}$ and $B_{2}$ out of both the $B_{1} C_{2}$ and $B_{2} C_{1}$ pairs, but they showed random choices with pairs $B_{1} B_{2}$ and $C_{1} C_{2}$. Table 2 shows that this is precisely the result simulated by Luce's model. Generally, we find that Luce's model yields reasonably good, but rarely excellent, matches with empirical transitive responding results. This is because this model (and other similar conditioning models) does not cope with the behaviour shown by some subjects, who perform well with training pairs but do badly with transitivity test pairs. Because of this, we will later propose modifications that improve the model's performance. However, before matters are complicated further, it is opportune to begin with the model's conversion into a neural network.

\section{NETWORKING LUCE'S MODEL}

As described, Luce's learning model manages to account quite well for a variety of empirical transitive results obtained with non-verbal syllogisms. 
TABLE 2

Percent Accuracy for Training and Test Pairs Observed for Transitive Subjects $(n=10)$ and as Simulated by Luce's Model (from Siemann, 1994a)

\begin{tabular}{lccccccc}
\hline & \multicolumn{3}{c}{ Training Pairs } & & \multicolumn{2}{c}{ Test Pairs } \\
\cline { 2 - 4 } \cline { 5 - 7 } & $\mathrm{AB}_{1}$ & $\mathrm{~B}_{1} \mathrm{C}_{1}$ & $\mathrm{C}_{1} \mathrm{D}$ & & & $\mathrm{B}_{1} \mathrm{~B}_{2}$ & $\mathrm{~B}_{1} \mathrm{C}_{2}$ \\
& $\mathrm{AB}_{2}$ & $\mathrm{~B}_{2} \mathrm{C}_{2}$ & $\mathrm{C}_{2} \mathrm{D}$ & & & $\mathrm{C}_{1} \mathrm{C}_{2}$ & $\mathrm{~B}_{2} \mathrm{C}_{1}$ \\
\hline Expected & 98 & 94 & 96 & & 50 & 94 \\
Observed & 98 & 96 & 91 & & 56 & 90 \\
\hline
\end{tabular}

It is reasonable to assume that the nervous system achieves transitive performances by mechanisms whose operations are described globally by this (or some similar) algebra. However, it is not immediately obvious how neurons might perform the necessary functions. Connectionist models have been shown to illustrate how conditioning processes-and, indeed, complex cognitive functions-might be realised in brain-like systems (e.g. Maki \& Abunawass, 1991; Shastri \& Ajjanaggade, 1993). We thus endeavoured to design a neural network that functions in a manner akin to that described by the algebraic model. Our assumption is that, in the long run, this kind of modelling might help to reveal some peculiarities of transitive responding as being due to constraints inherent to neuronal substrates (cf. Carmesin \& Schwegler, 1994).

After some unsuccessful attempts to adapt some of the currently fashionable networks, we were guided by the fact that, as applied here, Luce's model describes an instance of instrumental reinforcement learning. Adaptive neuronal networks operating on the basis of responsedependent reinforcement feedback were proposed quite early by Amari (1977). Perhaps because the crucial issue of allocation of connectivity updates was never dealt with specifically enough, this type of network, although successful in mimicking some psychologically interesting competencies, has been less popular than other kinds of networks (but see Donahoe, Burgos, \& Palmer, 1993). However, it will be shown that a particular network of this type is capable of transitive responding based on principles similar to those embodied in Luce's algebraic model.

The network consists in the main of an input layer $(j)$, a hidden layer $(k)$ and an output layer (l) (Fig. 2). Each layer contains the same number of units. That number must not be less than the number of different stimuli $(i)$ occurring in the premise or training pairs of a transitivity experiment, as the input units $j$ react specifically to these stimuli and the output units $l$ issue specific responses directed at the same stimuli. The output of a $j$ unit is $o_{j}=1$ if the corresponding stimulus $i$ is present and $\mathrm{o}_{j}=0$ if it is not. The input units are connected in a one-to-one fashion 
316

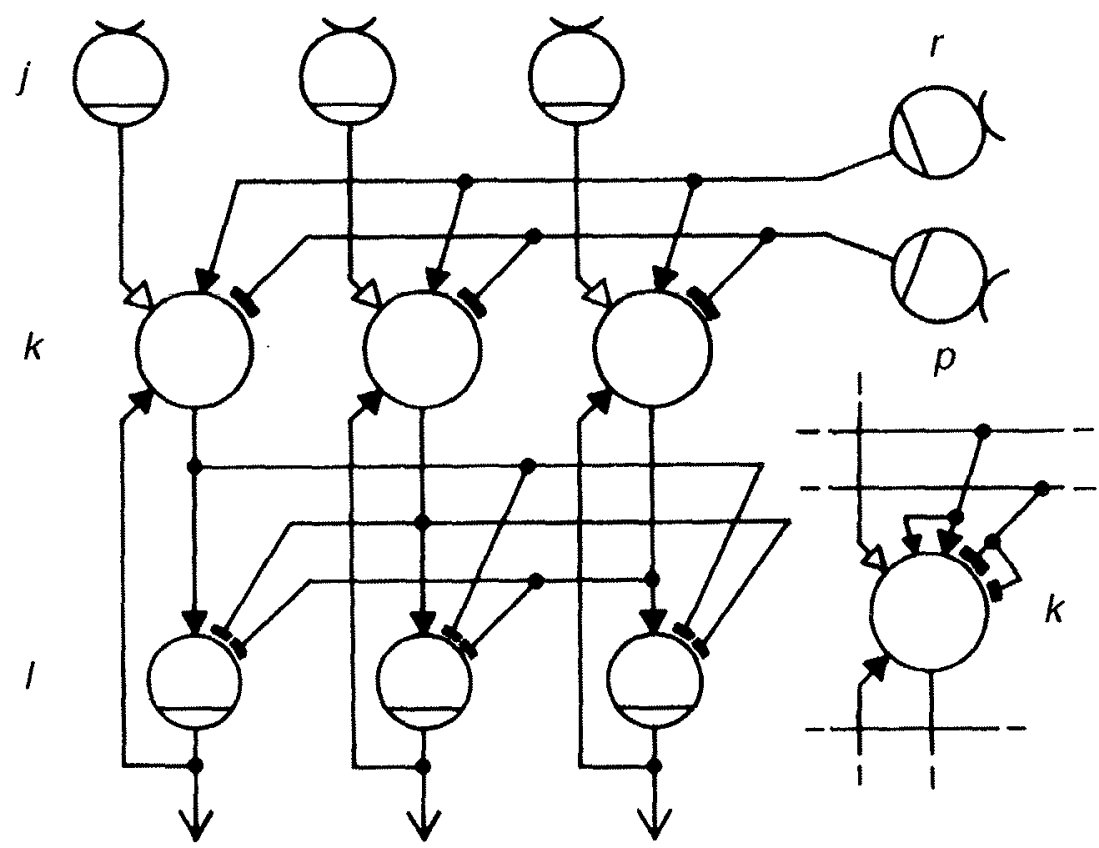

FIG. 2. Adaptive neuronal net capable of transitive responding based on principles similar to those embodied in Luce's leaning model. Only three exemplary units per input $(j)$, hidden $(k)$ and output $(l)$ layer are shown. The $r$ and $p$ units are concerned with the detection and signaling of response-contingent rewards and penalties. The fecdback connections from $/$ to $k$ are concerned with credit assignment control. Dishes indicate input units, arrows output units and bars units with thresholds. Triangles represent excitatory connections and arcs inhibitory connections. Empty triangles indicale variable comnections; dols indicate branching comnections. The $k$ insert is explained later in the text.

to the hidden units $k$. The activation of an intermediate unit $k$ due to the input from $j$ is directly $\mathrm{a}_{k}=o_{j} \times \mathrm{w}_{j k}$ with $j=k$ and where the all-important connection weight $w_{j k}$ will be specified soon. The activation results in an output $o_{k}=a_{k}$. However, the activation is also preserved temporarily buffered in the relevant $k$ units as a $c_{k}=a_{k}$ state. The significance of this latent state, and also of the other connections inputting into the $k$ units, will be explained below.

The output layer $l$ needs to behave according to a winner-takes-all principle to conform with the fact that subjects can choose only one of the stimuli in a given trial. It is traditional at this juncture to appeal to a lateral inhibition scheme without detailing it. For a reason that will become obvious shortly, we need to be more definite here. All intermediate units $k$ are connected to all output units $l$. The activation of $l$ units is directly $\mathrm{a}_{l}=\Sigma_{k} \mathrm{o}_{k} \times \mathrm{w}_{k l}$ with $\mathrm{w}_{k l}=1$ for $k=l$ and $\mathrm{w}_{k l}=-1$ for 
$k \neq l$. The output of $l$ units is determined by a threshold function where either $o_{l}=1$, a response to stimulus $i=l$ when $a_{l}>0$, or $o_{l}=0$, no response when $a_{l} \leqslant 0$. Thus, on a given trial, the lateral inhibition net yields the required single response to a single stimulus in a $l=i$ corresponding manner so long as it only has to deal with pairs of stimuli as is usual in standard transitivity designs.

However, this also holds for a pooled inhibition network modified from a flow-graph by McFarland (1965). Although slightly more complex, it demands fewer distant connections than the aforementioned network. It requires a single pooling unit $m$ and a series of $n$ units accompanying the $l$ units (Fig. 3). Given $w_{k m}=1$, the activation and output of the $m$ unit is $a_{m}=o_{m}=\Sigma_{k} o_{k}$. The activation of the $n$ units is directly $\mathrm{a}_{n}=\mathrm{o}_{k} \times \mathrm{w}_{k n}+\mathrm{o}_{m} \times \mathrm{w}_{m m}$, where $\mathrm{w}_{k n}=1$ and $\mathrm{w}_{m n}=-1$. The output of the $n$ units is $o_{n}=1$ if $a_{n}>0$ or $o_{n}=0$ otherwise. With $\mathrm{w}_{k l}=1$ and $\mathrm{w}_{n l}=1$, the $l$ units act like Boolean and-gates through an activation $a_{l}=o_{k} \times o_{n}$ and a threshold function $o_{l}=1$ when $a_{l}>0$ and $o_{l}=0$ when $a_{l} \leqslant 0$. This lateral inhibition network also yields a single response per

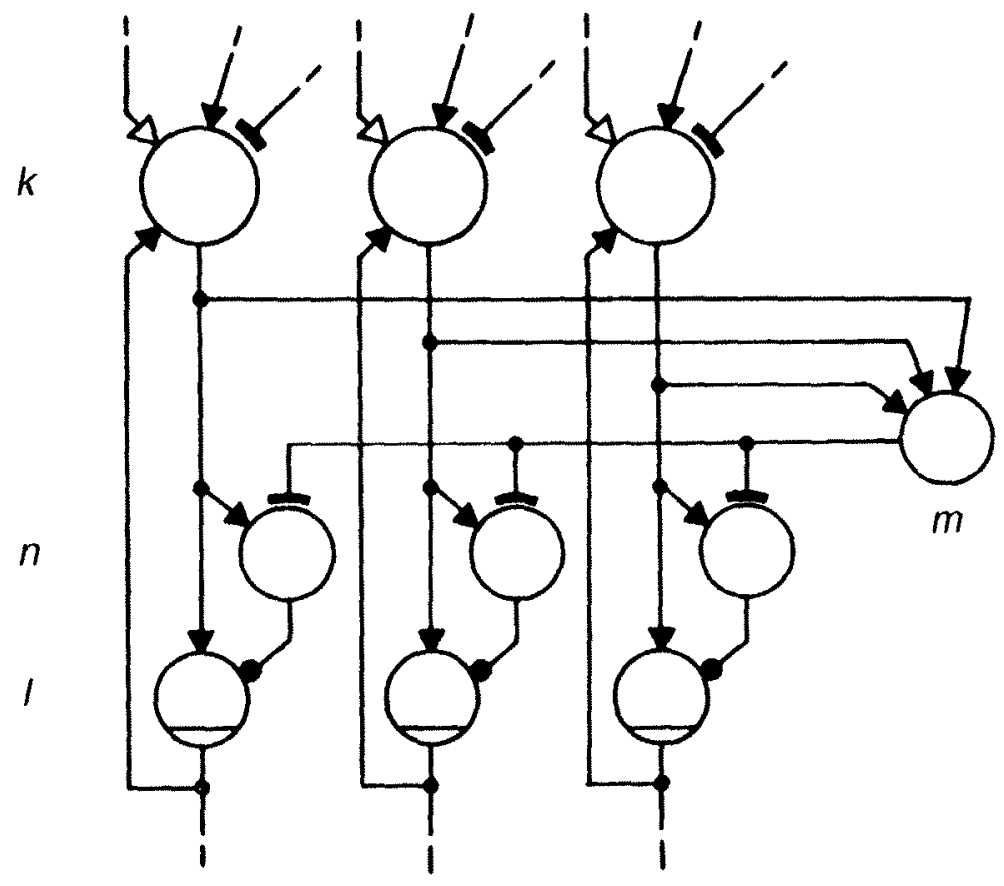

FIG. 3. Alternative lateral inhibition network based on a proposal by McFarland (1965). It involves an inhibition pooling unit $(m)$ and a layer of comparison units $(n)$. Solid circle terminals indicate multiplying connections. For other symbols, see legend to Fig. 2. 
trial, provided it is confronted only with pairs of stimuli. Other available network schemes can be used to ensure the winner-takes-all performance when the presentation of more than two stimuli is an experimental option, but the functioning of these alternative nets tends to be rather unintelligible because of the extra complexities they involve (Fukai \& Tanaka, 1997; McFarland, 1965; Yuang, Cohen, \& Kincaid, 1996).

Depending on whether the response issued corresponds to a stimulus scheduled to yield a reward or corresponds to a stimulus scheduled to yield a penalty, either input unit $r$ or input unit $p$ will be activated with the delay inherent to reinforcement delivery. We disregard here the effects of longer delays that are sometimes artificially instituted (see Sutton \& Barto, 1981). The reinforcement-sensitive units issue outputs according to $\mathrm{o}_{r}=1, \mathrm{o}_{p}=0$ or $\mathrm{o}_{p}=1, \mathrm{o}_{r}=0$, depending on whether the response produced by the $l$ unit results in the delivery of a reward or a penalty. These outputs are relayed to each of the intermediate layer units (Fig. 2). The arrangement is thought of as being analogous to the widely distributed systems signalling reward and punishment that are found in real brains (Rolls, 1975). The reward and penalty signals induce delayed states in the hidden $k$ units according to $\mathrm{f}_{k}=\mathrm{w}_{r k} \times \mathrm{o}_{r}$ and $\mathrm{g}_{k}=\mathrm{w}_{p k} \times \mathrm{o}_{p}$, where $\mathrm{w}_{r k}$ and $\mathrm{w}_{p k}$ are equivalent, respectively, to the parameters $\beta_{+}$and $\beta$. of Luce's algebraic model.

Luce's algebraic model implicitly assumes that the value increment or decrement due to reward or penalty is specifically apportioned to the actual stimulus-response link that brought about the particular reinforcement on a given occasion. The network must, of course, be explicit about this point. It solves the credit assignment problem by incorporating oneto-one feedback connections from the output units $l$ to the corresponding units $k$ (Fig. 2). These feedback connections excite units $k$ into a delayed state $\mathrm{e}_{k}=\mathrm{w}_{l k} \times \mathrm{o}_{l}$ with $\mathrm{w}_{l k}=1$, that is $\mathrm{e}_{k}=\mathrm{o}_{l}$. This state is $\mathrm{e}_{k}=1$ in the $k$ unit that corresponds $i=k$ to the stimulus $i$ that was reacted to, and $\mathrm{e}_{k}=0$ in all the $i \neq k$ units that correspond to the stimuli not reacted to. It effectively works as a gating factor. In conjunction with the latent state $c_{k}$ and the delayed states $\mathrm{f}_{k}$ or $\mathrm{g}_{k}$, specified earlier, it causes a Hebbianstyle weight update (Hebb, 1949; cf. Kandel, Schwartz, \& Jessell, 1991) of the specifically relevant $j$ to $k$ connection weight according to $w_{j k} \leftarrow$ $\mathrm{w}_{j k}+\mathrm{w}_{j k} \times \mathrm{c}_{k} \times \mathrm{e}_{k} \times \mathrm{f}_{k}-\mathrm{w}_{j k} \times \mathrm{c}_{k} \times \mathrm{e}_{k} \times \mathrm{g}_{k}$. Note that the latter state complex controls only the weight modification process and does not result in any actual output. Because of their multiplicative interaction, whenever any of the components $c_{k}, e_{k}, f_{k}$ or $g_{k}$ takes the value of zero, the weight increment or decrement part of the function also takes the value of zero. Only the $j$ to $k$ connection linking the stimulus to which the response is directed will undergo a weight change, provided that the experimenter has programmed it to yield a reward or a penalty, within 
the premise training. Such reinforcement allocation does not normally apply to the stimuli forming the transitivity test pairs, however. Furthermore, all the state components self-reset to zero as soon as the weight modification has taken place. Note also that the growth of the $w_{j k}$ connections is unbounded, much as the values $V_{x}$ are in Luce's model. The corpus of transitivity results so far available, as already remarked, does not appear to require the specification of stimulus value asymptotes and there is no evidence that the growth in synaptic effectiveness occurring in real nervous systems is limited in any specific way (Kandel et al., 1991). Nevertheless, a compromise against infinite growth could be that values and connectivities are incremented/decremented according to $V_{x} \leftarrow$ $V_{x} \pm f n\left(V_{x}\right) \times \beta$ and $w_{j k} \leftarrow w_{j k} \pm f n\left(w_{j k}\right) \times s_{k}$, where fn is a doubleexponential function of the form $y=\mathrm{e}^{-w^{-N}}, \beta$ stands for both reinforcement parameters, and $s_{k}$ stands summarily for either of the latent compound states mentioned above. This scheme yields more realistic looking learning curves but they do not affect critically the transitivity-producing properties of the model.

We believe that recursive connections like to the $l$ to $k$ links just described may be essential in any neural network expected to implement instrumental discriminations, or in a different terminology, operant stimulus response control. Moreover, they could be quite realistic, as feedback pathways are known to be frequent in real brains (Kandel et al., 1991). Perhaps they can be thought of as a kind of a neurally plausible replacement for the neurally unrealistic back-propagation scheme of many currently popular networks. Whether that is so or not, as specified here the network operates in much the same way as Luce's algebraic algorithm on which it is based. Starting from closely similar, small $w_{j k}$ weights, and exposed to the training pairs of a standard non-verbal transitivity task for a sufficient number of trials, it generates an orderly ranking of the weight magnitudes $w_{j k}$ across the $k$ units. This corresponds closely to the ranking of the $V_{x}$ stimulus values generated by the algebraic Luce model under analogous conditions. Trials with non-reinforced test pairs consisting of non-adjacent stimuli activate the corresponding units according to existing $\mathrm{w}_{j k}$ weights without altering them.

However, as it stands, the network model, depending on the precise $w_{r k}$ and $w_{p k}$ chosen, tends to exhibit a perfectly correct discrimination and transitivity performance very soon after training starts, because of the above-mentioned $w_{j k}$ ranking and the winner-takes-all behaviour of the lateral inhibition networks. This does not correspond well with either the persisting occasional error choices shown by real organisms or with the percentage choice outcomes of Luce's algebraic model. The mismatches have to do with the fact that the choice function of Luce's model is deliberately probabilistic and that the real neurons of organisms are not deter- 
ministic elements. Real neurons are known to be inherently noisy (Kandel et al., 1991). Obviously, the random noise element is missing from our neural network. In principle, it is difficult to decide what are the best fitting qualities and quantities of noise that need to be added to a deterministic model (cf. Luce, 1995). To cut an awkward matter short, we specify that the critical output of the hidden $k$ units, which effectively acts on the output $l$ units, is determined by the function $\mathrm{o}_{k} \leftarrow \mathrm{o}_{k}+\mathrm{o}_{k} \times \mathrm{rn}$, where $\mathrm{m}$ is a normally distributed random variable that has a mean of 0 , a standard deviation $\sigma$, and which is sampled anew for each $k$ unit and each trial. What this does is to inject noise, realistically acting at all levels of the network, in a summary manner only at the critical level. With a judicious choice of the $\sigma$ parameter, the network will produce a realistically imperfect discrimination and transitivity behaviour quite similar to that of Luce's algebraic model (Fig. 4).

\section{ADDING VALUE TRANSFER}

While as already stated Luce's algebraic model yields reasonably satisfactory simulations of real data, there is a matter on which it is somewhat deficient. It does not attend to a rather direct value transfer mechanism that could in part be responsible for transitive responding results. As already mentioned, Fersen et al. (1990) proposed a theory of transitive responding based precisely on such value transfer. These authors assumed that given the stimulus pair $\mathrm{X}+\mathrm{Y}$ - during training, a rewarded choice of $X$ would have the effect of sizeably incrementing its value $V_{x}$ but that some fraction of this value increment would be transferred on to the value $\mathrm{V}_{y}$ belonging to the not-chosen, but simultaneously present, stimulus $Y$. In its earliest version, the theory also envisaged that the choice of $Y$ would lead to some decrement of its value $V_{y}$ belonging to the not-chosen but present stimulus $X$. In the published, and much simplified, version of the theory, this second component was omitted (Fersen et al., 1991).

Regardless of this detail, and as already noted, the value transfer theory was severely criticised for appealing to an undocumented mechanism. Davis (1992b), however, noted that the postulated value transfer could be due to a classical conditioning process which is adventitious to the operant discrimination paradigm generally used in non-verbal syllogism experiments. As the subjects learn the task $X+Y-$, stimulus $X$ is increasingly chosen. Accordingly, stimulus $\mathrm{Y}$ changes from a stimulus initially associated with penalty into one preceding reward - that is, into a Pavlovian conditioned stimulus. To a much lesser extent, the instrumentally positive stimulus $\mathrm{X}$ can also act as a conditioned stimulus by 

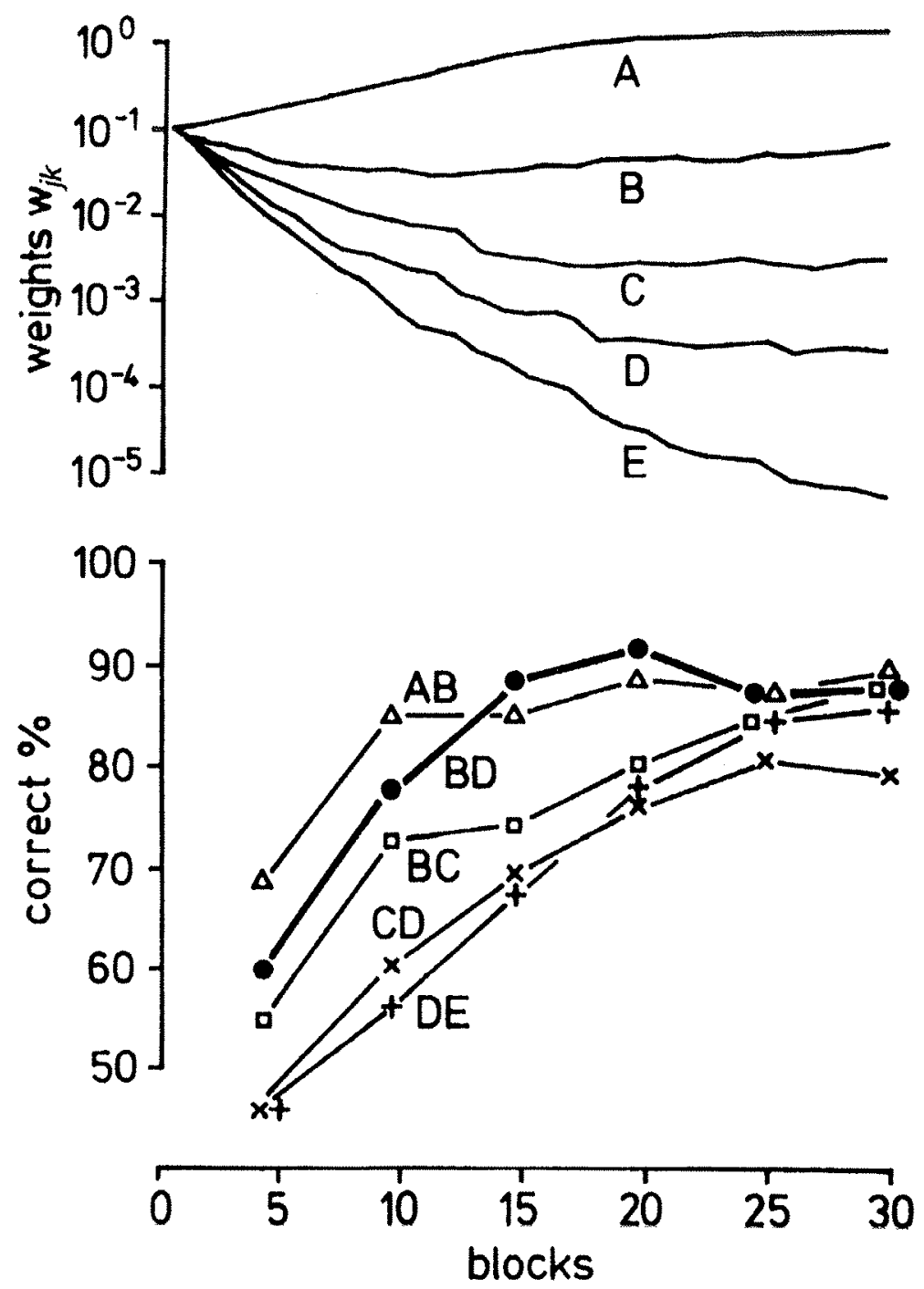

FIG. 4. Simulation of training (premise) pairs $(A+B-, B+C-, C+D-, D+E-$ ) learning and test (conclusion) pair (BD) performance in the Luce network. Connection weights $w_{j k}$ plotted as a function of trials (top) and percent correct choices (bottom) plotted as a function of blocks of five trials each (one per pair). Averages of 60 simulation runs with reinforcement weights $w_{, k}=0.2$ and $w_{p k}=0.7$, initial weight values $w_{j k}=0.1$, standard deviation $\sigma=0.8$ and $\min . / \max . x= \pm 1.5$. 
preceding the penalty that follows the responses to stimulus $Y$. Siemann, Daniel, Dombrowski and Delius (1993; see also Siemann, Delius, Dombrowski, \& Daniel, 1996) reported experimental support for a value transfer based on such a mechanism. They showed that stimuli accompanying operant discriminanda passively acquire response-eliciting properties proportional to the reinforcements consequent upon responses to the latter. In one of their experiments, pigeons were conditioned instrumentally to discriminate two target stimuli $(\mathrm{A}+, \mathrm{B}-)$ that were presented in random order on the same key. Each of these target stimuli was accompanied by a different neutral stimulus $\left(N_{a}\right.$ or $\left.N_{b}\right)$ displayed on another, nearby key. Responses to the latter stimuli had no scheduled consequences. When the discrimination of the target stimuli had been learned, $N_{a}$ and $N_{b}$ were presented paired together in unreinforced trials. As expected according to the value transfer idea, a clear preference for $N_{a}$ over $\mathrm{N}_{b}$ was observed. When the reinforcement allocations to the target stimuli were reversed, the preference for the satellite stimuli switched accordingly. Using a different design, Zentall and Sherburne (1994; cf. Zentall, Sherburne, Roper, \& Kraemer, 1996) demonstrated value transfer. using two concurrent simultaneous discriminations. During training, responses to the positive stimulus of one pair were always reinforced, whereas responses to the positive stimulus of the second pair were only sometimes reinforced. In tests, both the satellite stimuli were presented paired. As predicted by value transfer theory, the satellite stimulus that had accompanied the always reinforced stimulus was preferred. In yet another experiment, Steirn, Weaver and Zentall (1995; cf. Weaver, Steirn, \& Zentall, 1997) trained pigeons with the stimulus paris $\mathbf{A}+\mathbf{B}-, \mathbf{C}-\mathbf{E}+$, $\mathrm{C}+\mathrm{D}-$ and $\mathrm{A}+\mathrm{E}-$. Although these training pairs do not conform with a relational linear stimulus sequence, the pigeons still revealed a preference for $B$ in tests with the $B D$ pair in accordance to the value transfer principle. However, it is fair to say that there is still some disagreement about the extent of the role of value transfer in more standard transitivity experiments (Delius \& Siemann, 1998; Wynne 1995, 1997).

Luce's algebraic model can be adapted to meet the possibility that such transfer may intervene in transitivity tasks by specifying that, given $X+Y$-, besides the usual operant updates of the $X$ stimulus value $V_{x} \leftarrow$ $V_{x}+V_{x} \times \beta_{+} \times p_{x y}$ and the $Y$ stimulus value $V_{y} \leftarrow V_{y}-V_{y} \times \beta \times(1-$ $p_{x y}$ ), there are also parallel classical value updates for stimulus $Y$ and $X$ according to $V_{y} \leftarrow V_{y}+V_{y} \times \alpha_{+} \times p_{x y}$ and $V_{x} \leftarrow V_{x}-V_{x} \times \alpha \times\left(1-p_{x y}\right)$, respectively. The magnitudes of the classical learning parameters $\alpha^{+}$and $\alpha$ must, in practice, be a small fraction of the instrumental ones $\beta_{+}$and $\beta$, otherwise the adjunctive classical differentiation process begins seriously to interfere with the instrumental discrimination operation that is essential. Combining the classical and instrumental components, the 
overall updates are $\mathrm{V}_{\mathrm{x}} \leftarrow \mathrm{V}_{\mathrm{x}}+\mathrm{V}_{\mathrm{x}} \times \beta_{+} \times \mathrm{p}_{\mathrm{xy}}-\mathrm{V}_{\mathrm{x}} \times \alpha \times\left(1-\mathrm{p}_{\mathrm{xy}}\right)$ and $V_{y} \leftarrow V_{y}-V_{y} \times \beta_{-} \times\left(1-p_{x y}\right)+V_{y} \times \alpha_{+} \times p_{x y}$. A variant of this model, incorporating adaptations to their special design, yielded a good fit with the results of experiments aimed at demonstrating the existence of classically conditioned value transfer (Siemann et al., 1996).

While we were designing the network version of Luce's model described earlier, it unexpectedly emerged that it was virtually predestined to transfer value in a manner analogous to that proposed by Fersen et al. (1991). Note that to restrict the weight $\mathrm{w}_{j k}$ change to the $j$ to $k$ link that actually mediated the reinforcement, the network resorts to feedback gating through an $\mathrm{e}_{k}=\mathrm{o}_{l}$ state with $l=k=j$. We now retain that basic feature, but at the same time relax it by allowing an additional weight change affecting all the active $j$ to $k$ connections whether $\mathrm{e}_{k}$-gated or not according to the partial update $\mathrm{w}_{j k}^{\prime} \leftarrow \mathrm{w}_{j k}+\mathrm{w}_{j k} \times \mathrm{c}_{k} \times \mathrm{f}_{k}^{\prime}-\mathrm{w}_{j k} \times \mathrm{c}_{k} \times \mathrm{g}_{k}{ }_{k}$ with the states $\mathrm{f}_{k}^{\prime}=\mathrm{v}_{r k} \times \mathrm{o}_{r}$ and $\mathrm{g}^{\prime}{ }_{k}=\mathrm{v}_{p k} \times \mathrm{o}_{p}$ and where the weights $\mathrm{v}_{r k}$ and $v_{p k}$ are equivalent to the $\alpha_{+}$and $\alpha_{\ldots}$ parameters of the aforementioned algebraic model. Although other constructs are conceivable, the simplest is to assume that the new $\mathrm{v}_{r k}$ and $\mathrm{v}_{p k}$ weights correspond to doubled-up $r$ to $k$ and $p$ to $k$ connections (Fig. 2, insert $k$ ). In combination with the earlier instrumental weight change, this classically occasioned weight change will lead to a new overall weight update $\mathrm{w}_{j k} \leftarrow \mathrm{w}_{j k}+\mathrm{w}_{j k} \times \mathrm{c}_{k} \times \mathrm{e}_{k} \times \mathrm{f}_{k}$ $w_{j k} \times \mathrm{c}_{k} \times \mathrm{e}_{k} \times \mathrm{g}_{k}+\mathrm{w}_{j k} \times \mathrm{c}_{k} \times \mathrm{f}_{k}-w_{j k} \times \mathrm{c}_{k} \times \mathrm{g}_{k}^{\prime}$.

\section{CONFIGURING LUCE'S MODEL}

A further, important drawback of Luce's model, and indeed of all other conditioning models proposed so far (except one by Wynne, 1995), is that it fails to predict the regularly observed non-transitive performance of some experimental subjects, particularly human ones. These subjects perform very well with the premise pairs but do very poorly when faced with the conclusion pairs (Siemann, 1993a,b, 1994a,b; Siemann \& Delius, 1993; Werner et al., 1992). Such subjects thus appear to learn much about the separate training pairs, but little about the repeating elemental stimuli that constitute them. This behaviour seems to be the result of an alternative set that subjects can adopt and not to any stable individual disposition. Pigeons, however, seem less prone than human subjects to adopt the configural strategy except under special circumstances (Siemann \& Krug, 1996; see also below).

In Luce's model (and other similar models), each stimulus is subject to positive as well as to negative value updatings, depending on the kind of reinforcement that follows a choice response. As explained earlier, as soon as the training pairs are responded to at a better-than-chance level, 
the model inevitably yields transitive choices with unreinforced test pairs. A model capable of non-transitive test performance, even after good training pair performance has been attained, must thus incorporate some kind of stimulus value dissociation capable of overcoming the above link (Siemann, 1993a; Wynne, 1995). The $\varepsilon \kappa$ modification of Luce's model proposed by Siemann (1994a) adapts a notion already well established in the context of classical conditioning. It is the assumption that a given cue can separately gain or lose in associative strength through either being an individual stimulus or being a part of a compound stimulus (cf. Pearce, 1987; Rescorla, 1973). According to the $\varepsilon \kappa$ model, each stimulus is assumed to have two values, an elemental one and a configural one, within each stimulus pair. Given the pair $X+Y-$, the value of the elemental stimulus $X$ is updated according to $V_{x} \leftarrow V_{x}+V_{x} x$ $\beta_{+} \times p_{x y} \times \varepsilon$, while the value of elemental stimulus $Y$ is updated according to $\mathrm{V}_{\mathrm{y}} \leftarrow \mathrm{V}_{\mathrm{y}}-\mathrm{V}_{\mathrm{y}} \times \boldsymbol{\beta} \times\left(1-\mathrm{p}_{\mathrm{xy}}\right) \times \varepsilon$. The parameter $\varepsilon$ determines the weight of these elemental value changes. At the same time, the value of stimulus $X$ configured with $Y$ is updated in accordance with $V_{x(y)} \leftarrow V_{x(y)}+V_{x(y)} \times \beta_{+} \times p_{x y} \times \kappa$, while the value of stimulus $Y$ configured with $X$ is updated in accordance with $V_{y(x)} \leftarrow V_{y(x)}-V_{y(x)} \times \beta \times(1-$ $\left.p_{x y}\right) \times \kappa$, where for convenience we have introduced a dummy parameter $\kappa=1-\varepsilon$.

The elemental value of a stimulus is naturally also subject to update when that same stimulus is a constituent of another training pair, as for example $V_{y}$ when stimulus $\mathrm{Y}$ occurs within the $\mathrm{Y}+\mathrm{Z}-$ pair. However, within this same pair, it is the alternative configural value of $Y$, namely $V_{y(z)}$ and naturally not $V_{y(x)}$, that is subject to updating. The choice probability for the stimuli constituting a training pair $\mathrm{X}+\mathrm{Y}$ - is computed by the products of the relevant elemental and configural values according to $p_{x y}=\left(V_{x} \times V_{x(y)}\right) /\left(V_{x} \times V_{x(y)}+V_{y} \times V_{y(x)}\right)$. The multiplicative, rather than additive combination of values is preferred for the simple reason that it yields better fitting simulations of real data. Configural values are obviously not effective in test pairs, as these consist of stimuli that have never been presented paired together during training. The choice probability for an $\mathrm{XZ}$ test pair thus practically reduces to $\mathrm{p}_{\mathrm{xz}}=\mathrm{V}_{\mathrm{x}}$ / $\left(V_{x}+V_{z}\right)$, since both $V_{x(y)}$ and $V_{y(x)}$ retain whatever identical initial positive value they were given.

The effect that variations of the weighting parameter $\varepsilon$ has on the performance of this model is illustrated in Fig. 5. With $\varepsilon=0$ (and hence $\kappa=1$ ), the model yields perfect training pair accuracies but a chance-level test pair performance. Larger $\varepsilon$ values yield increasingly better transitivity without appreciably affecting training pair accuracy. With $\varepsilon=1$ (and hence $\kappa=0$ ), the $\varepsilon \kappa$ model behaves much as Luce's original model, exhibiting among other things a symbolic distance effect. This effect refers to the fact that the 


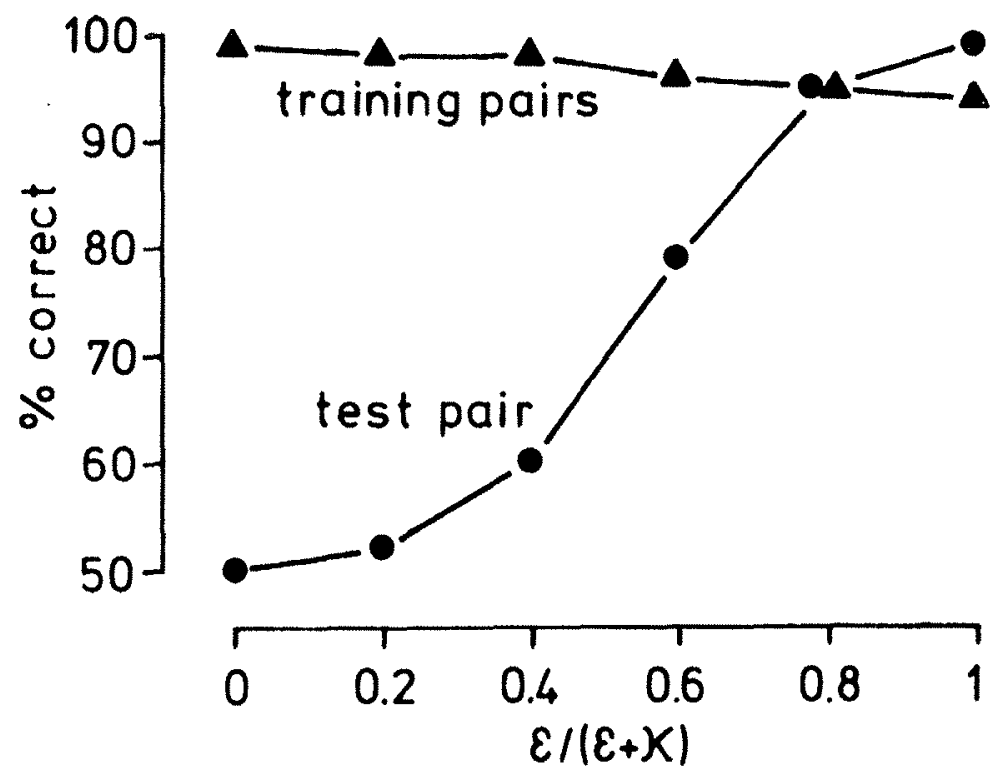

FIG. 5. Effects of varying the $\varepsilon / K$ ratio on the transitivity training and test performance of the $\varepsilon \kappa$ model. Final averaged accuracy for pooled $A+B-, B+C-, C+D-$ and $D+E-$ training pairs and for test pair $\mathrm{BD}$. Averages of 10 simulation runs, each lasting 30 trials with leaming parameters $\beta_{+}=0.65$ and $\beta_{-.}=0.95$.

choice accuracy with pairs composed of stimuli further apart in the implicit series tends to be empirically better than with pairs composed of stimuli close together in that same series (Breslow, 1981).

\section{PERFORMANCE OF THE $\varepsilon \kappa$ MODEL}

The superiority of the $\varepsilon \kappa$ model over Luce's original model is supported by many empirical results. For example, in one transitive responding experiment, human adults were trained with the usual overlapping pairs of adjacent stimuli corresponding to a linear $\mathrm{A}+$ to $\mathrm{F}$ - series and subsequently tested with the pairs of non-adjacent stimuli $\mathrm{BD}, \mathrm{BE}$ and $\mathrm{CE}$ (Siemann, 1994a). For the transitively responding subjects, the simulated and observed error rates were nearly identical; for non-transitively responding subjects, they were quite close (Table 3). Note that Luce's model-and, in fact, all the other conditioning models that have been proposed to account for transitive responding-plainly fail to predict non-transitive test performance whenever the training pairs have been successfully discriminated (but see Wynne, 1995). 
TABLE 3

Observed and Simulated ( $\varepsilon \kappa$ Model) Percent Accuracies for Transitive $\left(n=8 ; \beta_{+}=0.05\right.$, $\beta_{-}=0.30$; Linear: $\varepsilon=0.85$; Circular: $\left.\varepsilon=0.55\right)$ and Non-transitive $\left(n=8 ; \beta_{+}=0.01, \beta_{-}=0.10\right.$; Linear: $\varepsilon=0.05$; Circular: $\varepsilon=0.45$ ) Human Subjects for a Linear Task and a Circular Task (from Siemann, 1994a)

\begin{tabular}{|c|c|c|c|c|c|c|c|c|c|}
\hline & & & Traini & Pairs & & & & Pair & \\
\hline Linear task & & & & & & & & & \\
\hline Transitive & $\mathrm{AB}$ & $\mathrm{BC}$ & $\mathrm{CD}$ & $\mathrm{DE}$ & $\mathrm{EF}$ & & $B D$ & $B E$ & $C E$ \\
\hline simulated & 98 & 95 & 93 & 91 & 96 & & 95 & 98 & 88 \\
\hline observed & 97 & 95 & 92 & 92 & 97 & & 92 & 95 & 89 \\
\hline Non-transitive & $A B$ & $\mathrm{BC}$ & $\mathrm{CD}$ & DE & $\mathrm{EF}$ & & $B D$ & $\mathrm{BE}$ & $\mathrm{CE}$ \\
\hline simulated & 93 & 92 & 92 & 92 & 92 & & 50 & 50 & 50 \\
\hline observed & 95 & 92 & 90 & 87 & 90 & & 46 & 34 & 59 \\
\hline Circular task & & & & & & & & & \\
\hline Transitive & $\mathrm{AB}$ & $\mathrm{BC}$ & $\mathrm{CD}$ & $\mathrm{DE}$ & $\mathrm{EF}$ & FA & $B D$ & $B E$ & $\mathrm{CE}$ \\
\hline simulated & 81 & 93 & 97 & 96 & 95 & 99 & 84 & 91 & 88 \\
\hline observed & 92 & 97 & 94 & 94 & 94 & 93 & 80 & 85 & 67 \\
\hline Non-transitive & $A B$ & $\mathrm{BC}$ & $\mathrm{CD}$ & $\mathrm{DE}$ & $\mathrm{EF}$ & FA & $\mathrm{BD}$ & $B E$ & $\mathrm{CE}$ \\
\hline simulated & 90 & 94 & 95 & 95 & 93 & 94 & 49 & 49 & 50 \\
\hline observed & 95 & 92 & 96 & 96 & 92 & 93 & 46 & 40 & 43 \\
\hline
\end{tabular}

Although they probably do not do so as readily as humans, pigeons also occasionally show non-transitive responding. Higa-King and Staddon (1993) trained pigeons on a $\mathrm{A}+$ to $\mathrm{E}-$ linear series using two rather special procedures. The birds were repeatedly presented with either the currently least-well discriminated stimulus pair (response-based procedure) or with the currently least-experienced stimulus pair (time-based procedure). The procedures were unevenly successful in ensuring a good training pair discrimination but, when they did so, the pigeons sometimes failed to yield transitivity with the BD test pair. This can be attributed to a predominant configural learning of the training pairs. It is conceivable that the serially repeated presentation of the various training pairs associated with the procedures used sometimes promoted configural learning. The $\varepsilon \kappa$ model has no difficulty with matching these results if $\varepsilon$ (and thus $\kappa$ ) is allowed to vary, depending on the precise conditions. Useful simulations, however, require more details about the precise course of the experiments than are provided in the relevant paper.

Fersen et al. (1991) let pigeons learn the overlapping adjacent stimulus pairs of a $\mathrm{A}+$ to $\mathrm{G}-$ linear series. The pigeons then responded transitively to test pairs of non-adjacent stimuli. A pair $\mathrm{G}+\mathrm{A}$ - was subsequently added to the training set, closing the series to a circle. Performance on all pairs dropped to chance level to begin with, but two pigeons out of four eventually managed to (re)learn all training pairs. 
However, when tested again with test pairs composed of non-adjacent stimuli, they chose randomly. This agrees with a switch from elemental to configural learning as predicted by the $\varepsilon \kappa$ model. With humans, Siemann (1994a) found that most of their subjects ( 7 of 8$)$ who had responded transitively to test pairs after learning a linear series $\mathrm{A}+$ to $\mathrm{F}$ - also continued to do so when the task was converted to a circular one by training with a $\mathrm{F}+\mathrm{A}-$ pair. These subjects appeared to have coded the freak pair as a configural exception, indicating that humans may also be able to bring to bear locally different $\varepsilon$ and $\kappa$ values, an issue that is not pursued further here. The remaining subjects $(n=8)$ also learned both tasks but always responded non-transitively to test pairs of non-adjacent stimuli. These subjects appeared to have learned all training pairs configurally. Both these results were accommodated by the $\varepsilon \kappa$ model with a suitable parameter adjustment (Table 3 ). Note that, because intermediate $\varepsilon$ and $\kappa$ magnitudes are being used within a population model, they reflect mainly a mixture of individuals learning according to either a predominantly elemental or to a predominantly configural mode.

Siemann and Delius (1994) similarly trained a group of 10 humans and a group of 3 pigeons with all the 10 pairs that can be assembled with five stimuli with the reinforcement allocations to the stimuli in consonance with a circular structure $(\mathrm{A}+\mathrm{B}-, \mathrm{B}+\mathrm{C}-, \mathrm{C}+\mathrm{D}-, \mathrm{D}+\mathrm{E}-, \mathrm{A}+\mathrm{C}-, \mathrm{D}+\mathrm{A}-$, $\mathrm{E}+\mathrm{B}-, \mathrm{B}+\mathrm{D}-, \mathrm{E}+\mathrm{A}-$ and $\mathrm{C}+\mathrm{E}-)$. The human subjects managed to learn this task but the pigeons did not. The human results were simulated by the purely configural version of the $\varepsilon \kappa$ model $(\varepsilon=0, \kappa=1)$, yielding an accuracy of $94 \%$ mean correct responses over all 10 stimulus pairs. The simulated scores coincided closely with those observed empirically. The pigeon results of nearly random choices on all pairs are, of course, easily simulated with any of the elemental stimulus learning models (including Luce's model and the corresponding version of the $\varepsilon \mathrm{K}$ model).

The EK model also accounts for the results of experiments in which subjects learned two totally, or two partially, separate linear series and were later tested with stimulus pairs bridging these disparate series. While subjects who responded transitively to within-series test pairs consistently chose the higher-valued stimuli of the between-series test pairs (elemental strategy), subjects who failed to respond transitively in the first type of test also showed random responding in the second type of test (configural strategy; Werner et al., 1992).

Occasionally, human subjects respond with definitely anti-transitive choices to test pairs after training with a linear series, for example preferring, after training with a $\mathrm{A}+$ to $\mathrm{E}$ - series, the $\mathrm{D}$ stimulus to the $\mathrm{B}$ stimulus during tests (Siemann, 1993a). Within certain limits, the $\varepsilon \mathrm{K}$ model can also simulate this counterintuitive result. With an intermediate $\varepsilon$, a large $\beta_{+}$and a small $\beta .\left(\varepsilon=0.65, \kappa=0.35, \beta_{+}=0.99, \beta=0.01\right)$, it 


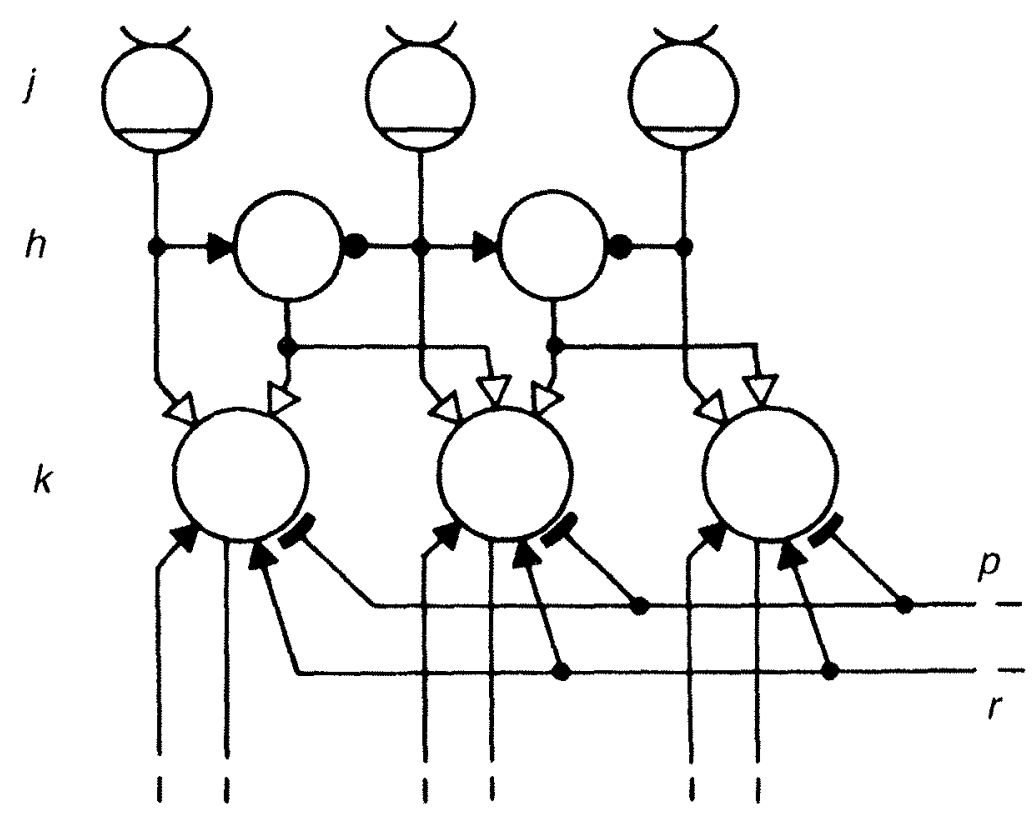

FIG. 6. Additional circuitry used to convert Luce's network into the ex network capable of both transitive and non-transitive responding. For explanation of the symbols, see legend to Fig. 2.

yields a simulated average training pair performance of $99 \%$ and an inverse, anti-transitive BD test pair performance of $33 \%$ correct choices.

To confer on the neural network of Fig. 2 the essential characteristics of the ek model, it is necessary to incorporate an additional layer of as many units as there are paired stimulus configurations (Fig. 6). Each of these $h$ interunits receives collateral connections from input units $j$ and $j+1$. Their activity and output is directly defined by $\mathrm{a}_{h}=\mathrm{o}_{h}=\mathrm{o}_{j} \times \mathrm{w}_{j h} \times$ $o_{j+1, h} \times w_{j+1, h}$. They effectively act as Boolean and-gates, their output only attaining the on-state $\mathrm{o}_{m}=1$ when the corresponding $i$ plus $i+1$ stimulus configuration is present. Each of the interunits $h$ connects with intermediate units $k$ and $k+1$ with a variable connection $w_{l / k}$. In conjunction with $o_{h}$, they directly generate a $\mathrm{d}_{k}$ latent state according to $\mathrm{d}_{k}=o_{h} \times \mathrm{w}_{h k}$. The weights of these $h$ to $k$ connections change in a Hebbian-like manner according to $\mathrm{w}_{h k} \leftarrow \mathrm{w}_{h k}+\mathrm{w}_{h k} \times \kappa \times \mathrm{d}_{k} \times \mathrm{e}_{k} \times \mathrm{f}_{k}-$ $\mathrm{w}_{j k} \times \kappa \times \mathrm{d}_{k} \times \mathrm{e}_{k} \times \mathrm{g}_{k}$. The weights of the direct $j$ to $k$ connections change as before according to $\mathrm{w}_{j k} \leftarrow \mathrm{w}_{j k}+\varepsilon \times \mathrm{w}_{j k} \times \mathrm{c}_{k} \times \mathrm{e}_{k} \times \mathrm{f}_{k-}$ $\varepsilon \times \mathrm{w}_{j k} \times c_{k} \times \mathrm{e}_{k} \times \mathrm{g}_{k}$. The activity and output of the $k$ layer units is now directly $\mathrm{a}^{\prime}{ }_{k}=\mathrm{o}^{\prime}{ }_{k}=\mathrm{o}_{j} \times \mathrm{w}_{j k}+\mathrm{o}_{h l} \times \mathrm{w}_{h k}$. In this manner, both an elemental and a configural input originating from a given stimulus 
converges on each response-mediating hidden unit. The different magnitudes of the algebraic model's $\varepsilon$ and $\kappa$ parameters can be thought of as being realised in the network by varying the physical distances between the two stimulus inputs from $j$ and from $h$ and the reinforcement inputs from $r$ and from $p$ upon the intermediate units $k$. The magnitude of synaptic changes can be realistically assumed to decrease as the anatomical distance between the relevant synapses increases (Kandel et al., 1991). Simulations with this model tend to exhibit either a predominantly elemental or a predominantly configural mode of learning. This may be modelling the tendency of both humans and animals to fall into one or the other kind of performance category. However, the $\varepsilon \kappa$ network opens many new perspectives that require further exploration.

\section{CONCLUSION}

We have shown that Luce's conditioning model, which is quite successful in simulating transitivity learning and responding, can be translated into a parallel-operating neural network of the reinforcement type. The exercise was not quite as trivial as we had initially imagined. Certain processing details that are simply assumed in the algebraic version of the model turned out to require accurate specification in the network version. For example, the apportioning of the learning effect to the correct stimulus-response link necessitates an unforeseen feedback connectivity. Because of that, the winner-takes-all output needs a more detailed specification than an accompanying line of text that is sufficient with the algebraic version of the model. When all is considered, however, the results of simulations with Luce's network are in close agreement with the behaviour of the algebraic model and thus in reasonably close agreement with that of transitively responding humans and animals. We have also described a minor addition to the model that makes it sensitive to a process of value transfer through classical conditioning, which, according to some recent evidence, probably contributes to the transitive responding in addition to instrumental conditioning.

A modified, more powerful $\varepsilon \kappa$ model that we propose, which allows for both elemental and configural learning about stimuli, additionally copes with the behaviour of weakly or not transitively responding individuals. It requires an additional interunit layer at the input side of the network. Note that the $\varepsilon \kappa$ modification only addresses the distinct elemental and compound stimulus valuation in the constrained context of transitivity learning and responding. At this stage of development, it is not meant to deal with the wider, more general issue of elemental versus configural stimulus processing in classical conditioning contexts (cf. Pearce, 1987; 
Rescorla \& Wagner, 1972). It is already clear, however, that the connectivities of the $\varepsilon \kappa$ network, once it has been suitably trained, agree well with an information-theoretical conclusion drawn by Carmesin and Schwegler (1994). They found that, among all the conceivable relational structures that a set of stimuli can assume through reinforcement inequalities, the hierarchically linear is precisely the structure that can be represented with the least connectivity complexity by a neural network. That is also in agreement with the empirical finding that humans and animals indeed require the least training to master such a structure (Siemann \& Delius, 1994). A linear hierarchical structure in turn is the only one that embodies consistent transitivity among all its constituent stimuli.

It is important to note that our network models have so far been deliberately designed to mimic the behaviour of the algebraic models as closely as possible and thus cannot yet be expected to yield any substantially different, or particularly startling, predictions. Rather, at this stage, the networks should be viewed as models that make more transparent the information flow and processing in neuronal structures designed to implement the algebraic models. Whatever the shortcomings of neural networks, they are bound to be more similar to the nervous system than the abstract symbolic operations of algebra. Indeed, our networking efforts make it clear that a probabilistic off-line choice function, which is almost trivial when algebraically expressed, turns out to be a more involved affair when networked to operate on-line. As we continue to develop these networks, it may well turn out that other choice functions yield simpler connectivities and perhaps even more accurate simulations.

We believe that the weight modification scheme imposed by the coincidence of both reinforcement and recursive feedback onto a hidden layer is an important feature in our network. It is also fair to point out that the network models presented here have occasionally been criticised for introducing an undesirable complexity by appealing to several different, non-standardised neural elements. However, real nervous systems are clearly much less parsimonious in this respect (Glanzman, 1995; Kandel et al., 1991). We must also assume that, in reality, far more neurons than envisaged in our networks are involved in bringing about the performances that interest us. Elsewhere we have estimated that a single peck of a pigeon to a specific stimulus may involve the activation of perhaps as many as $10^{5}$ neurons. A single human finger press on a key in response to a similar stimulus is likely to involve at least 10 times as many neurons. The information-processing options that can be associated with neural networks of such scale are nearly unimaginable and possibly beyond a thorough rational analysis.

By distinguishing between transitive responding and transitive inference, we have tried to underline that we are not claiming to have offered an 
algebraic or network model capable of explaining the solution of traditional verbal transitive syllogisms ("Anna is bigger than Paul", etc.; Breslow, 1981). However, the reader should consider whether operations akin to those postulated with the conditioning models might not occur in working memory as humans attempt to solve such syllogisms. It is worth stressing that the solution of a classical three-term syllogism is a really trivial operation. The premises " $\mathrm{A}$ is bigger than $\mathrm{B}$ " and " $\mathrm{B}$ is bigger than $C$ " translate easily into the propositions " $\mathrm{A}$ is big, $\mathrm{B}$ is small" and " $\mathrm{B}$ is big, $\mathrm{C}$ is small". The question "Is A bigger than C?" can be solved by simply referring to the label " $\mathrm{A}$ is big" (or " $\mathrm{C}$ is small") without recourse to any kind of deduction. Only syllogisms with five or more items challenge proper reasoning. But then even human adults are usually stumped by the question "Is Ben brighter than Diana?" after having been told once that "Ben is brighter than Carl, Emma is brighter than Diana, Anna is brighter than Ben, Carl is brighter than Diana", presumably because the information imparted exceeds their working memory capacities. Most subjects require repeated experience with the premises before they can conclude correctly with any assurance (Siemann \& Gebhardt, unpublished; see also Woocher, Glass, \& Holyoak, 1978). The memorisation of the premises requires rehearsal operations that could well have similarities with the cumulative conditioning processes involved in the models discussed here in connection with the solution of non-verbal syllogisms.

\section{REFERENCES}

Amari, S. (1977). A mathematical approach to neural systems. In J. Metzler (Ed.), Systems neuroscience, pp. 67-117. New York: Academic Press.

Boysen, S.T., Berntson, G.G., Shreyer, T.A., \& Quigley, K.S. (1993). Processing of ordinarity and transitivity by chimpanzees. Joumal of Comparative Psychology, 107, 208-215.

Breslow, L. (1981). Reevaluation of the literature on development of transitive inferences. Psychological Bulletin, 89, 325-351.

Bryant, P.E., \& Trabasso, T. (1971). Transitive inferences and memory in young children. Nature, 232, 456 458 .

Bush, R.R., \& Mosteller, F. (1951). A mathematical model for simple learning. Psychological Review, 38, 313 323.

Carmesin, H.O., \& Schwegler, H. (1994), Serial versus sequential processing of relational stimulus structures. Biological Cybernetics, 71, 523-529.

Chalmers, M., \& McGonigle, B. (1984). Are children any more logical than monkeys on the five-term series problem? Journal of Experimental Child Psychology', 37, 355-377.

Couvillon, P.A., \& Bitterman, M.E. (1992). A conventional conditioning analysis of "transitive inference". Journal of Experimental Psychology": Animat Behavior Processes, 18, 308-310. 
Davies, D.G.S., Staddon, J.E.R., Machado, A., \& Palmer, R.G. (1993). The process of recurrent choice. Psychological Review, $100,310-320$.

Davis, H. (1992a). Thansitive inference in rats. Joumal of Comparative Psychology, 106, 342-349.

Davis, H. (1992b). Logical transitivity in animals. In W.K. Honig \& J.G. Fetterman (Eds), Cognitive aspects of stimulus control, pp. 405-429. Hillsdale, NJ: Lawrence Erlbaum Associates Inc.

Delius, J.D., \& Siemamn, M. (1998). Transitive responding in animals and humans: Exaptation rather than adaptation? Beharioural Processes, 42, 107-137.

DeSoto, C.B., London, M., \& Handel, S. (1965), Social reasoning and spatial paralogic. Jounal of Personality and Social Psychology, 2, 513--521.

Donahoc, J.W., Burgos, J.E., \& Palmer, D.C. (1993). A selectionist approach to reinforcement. Journal of the Experintental Analysis of Behavion, 60, 17-40.

Evans, J.S.B.T., Newstead, S.E., \& Byrne, R.M.J. (1993). Human reasoning: The psychology' of deduction. Hove: Lawrence Erlbaum Associates Lid.

Fersen, L. von, Wynne, C.D.L., Delius, J.D., \& Staddon, J.E.R. (1990). Deductive reasoning in pigeons. Naturwissenschafion, 77, 548-549.

Fersen, L. von, Wyme, C.D.L., Delius, J.D., \& Staddon, J.E.R. (1991). Transitive inference formation in pigeons. Journal of Experimental Psychology: Animal Behavior Processes, 17, $334-341$.

Fukai, T., \& Tanaka, S. (1997). A simple neural network exhibiting selective activation of neuronal ensembles from winner-take-all to winner-share-all. Neural Computation, 9, 77-97.

Gillan, D.J. (1981). Reasoning in the chimpanze: Il. Transitive inference. Journal of Experimental Psychology: Animal Behavior Processes, 7, 150-164.

Glanzman, D.L. (1995). The cellular basis of classical conditioning in Aplysia californica: It is less simple than you think. Trends in the Newrosciences, 18, 30-35.

Harris, M., \& McGonigle, B. (1994). A model of tansitive inference. Quarterly Journal of Experimental Psychology, 47B, 310-348.

Hebb, D.O. (1949). The organization of behavior: A metropsychological theory. New York: John Wiley.

Higa-King, J.J., \& Staddon, J.E.R. (1993). "Transitive inference" in multiple conditional discriminations. Joural of the Experimental Analysis of Behavior, 59, 265291.

Johnson-Laird, P.N. (1986). Reasoning without logic. In T. Myers, K. Brown, \& B. McGonigle (Eds), Reasoning and discourse processes, pp. 13-49. London: Academic Press.

Kandel, E.R., Schwartz, J.H., \& Jessell, T.M. (Eds) (1991). Principles of neural science, 3rd edn. London: Prentice-Hall.

Luce, R.D. (1959). Individual choice behavior. New York: John Wilcy.

Luce, R.D. (1995). Four tensions concerning mathematical modeling in psychology. Anuual Review of Psychology, 46, 1-26.

Maki, W.S., \& Abunawass, A.M. (1991). A connectionist approach to conditional discriminations: Learning, short-term memory, and attention. In M.L. Commons, S. Grossberg, \& J.E.R. Staddon (Eds), Neural netw'ork models of conditioning and action, pp. 241 ... 278. Hillsdale, NJ: Lawrence Erlbaum Associates Inc.

Markovits, H., \& Dumas, C. (1992). Can pigeons really make transitive inferences? Jourhal of Experimemal Psychology: Animal Behavior Processes, 18, 311-312.

McFarland, D.J. (1965). Flow graph representation of motivational systems. British Journal of Mathematical and Statistical Psychology, 18, 25-43.

McGonigle, B., \& Chalmers, M. (1977). Are monkeys logical? Natme, 267, 694-696.

McGonigle, B., \& Chalmers, M. (1992). Monkeys arc rational! Quarterly Joumal of ExperiInental Psychology, 45B, 189-228. 
Pearce, J.M. (1987). A model for stimulus generalization in Paviovian conditioning. Psychological Review, 94, 61-73.

Piaget, J. (1921). Une forme verbale de la comparison chez l'enfant (A form of verbal comparisons in the child). Archives de Psychologie, 1921, $141 \cdots 172$.

Piaget, J. (1953). Logic and psychology. Manchester: Manchester University Press.

Rescorla, R.A. (1973). Evidence for "unique stimulus" account of configural conditioning. Journal of Comparative and Physiological Psychology', 85, 331-338.

Rescorla, R.A., \& Wagner, A.R. (1972). A theory of Pavlovian conditioning: Variations of the effectiveness of reinforcement and nonreinforcement. In A.H. Black \& W.F. Prokasy (Eds), Classical conditioning: Current research and theory, pp. 64 99. New York: Appleton.

Roberts, W.A., \& Phelps, M.T. (1994). Transitive inference in rats: A test of the spatial coding hypothesis. Psychological Science, 5, 368-374.

Rolls, E.T. (1975). The brain and reweard. Oxford: Pergamon Press.

Shastri, L., \& Ajjanaggade, V. (1993). From simple association to systematic reasoning: A connectionist representation of rules, variables and dymamic bindings using temporal synchrony. Behavioral and Brain Sciences, 16, 417-494.

Siemann, M. (1993a). Transitive Inferenz: Experimentelle Untersuchung einer kognitiven Leistung (Transitive inference: Experimental investigation of a cognitive competence). $\mathrm{PhD}$ thesis, Universität Konstanz.

Siemann, M. (1993b). "Transitive inferences" in pigeons and humans. In N. Elsner \& M. Heisenberg (Eds), Gene, brain and behavior, p. 857. Stuttgart: Thieme.

Siemann, M. (1994a). Uberprüfung einfacher Modelle zum transitiven Schlußfolgern bei nonverbaler Aufgabenform (Test of simple models of transitive deduction with nonverbal task forms). Zeitschrift fiit Experimentelle und Angen'andte Psychologie, 4l, 584-616.

Siemann, M. (1994b). Memory load influences on transitive responding in humans. In $N$. Elsner \& H. Breer (Eds), Sensory transfluction, p. 847. Stutgart: Thieme.

Siemann, M. (1996). Transitive Inferenz bei verbaler und nonverbaler Aufgabenstellung: Ein Überblick (Transitive inference with verbal and non-verbal task presentation: A review). Zeitschrift für Psychologic, 204, 233-259.

Siemam, M., Daniel, D., Dombrowski, D., \& Delius, J.D. (1993). Value transfer in pigeon conditioning. In N. Elsner \& M. Heisenberg (Eds), Gene, brain and behavior, p. 856. Stutgart: Thieme.

Siemann, M., \& Delius, J.D. (1993). Implicit deductive responding in humans, Natuuissenschaften, 80, 364-366.

Siemann, M., \& Delius, J.D. (1994). Processing of hierarchic stimulus structures has advantages in humans and animals. Biological Cybernetics, 71, 531-536.

Siemann, M., \& Delius, J.D. (1996). Influences of task concreteness upon transitive responding in humans. Psychological Research, 59, $81-93$.

Siemann, M., Delius, J,D., Dombrowski, D., \& Daniel, S. (1996). Value transfer in pigeon discrimination learning. Psychological Record, 46, 707--728.

Siemann, M., Delits, J.D., \& Wright, A.A. (1996). Transitive responding in pigeons: Influences of stimulus frequency and reinforcement history. Behavioural Processes, 37, 185 195,

Siemann, M., \& Krug, I. (1996). Further evidence for a value transfer in pigcons. In N. Elsner \& H.U. Schnitzler (Eds), Proceedings of the Göttingen Neurobiology Meeting, p. 782. Stuttgart: Thieme.

Steirn, J.N., Weaver, J.E., \& Zentall, T.T. (1995). Transitive inference in pigeons: Simplified procedures and a test of value transfer theory. Animal Leaning and Behavior, 23, 76-82.

Sutton, R.S., \& Barto, A.G. (1981). Toward a modern theory of adaptive networks: Expectation and prediction. Psychological Revien', 88, 135-170. 


\section{4}

Trabasso, T., \& Riley, C.A. (1975). The construction and use of representations involving linear order. In R. Solso (Ed.), Information processing and cognition, pp. 381-410. New York: Lawrence Erlbaum Associates Inc.

Treichler, F.R., \& Van Tilburg, D. (1996). Concurrent conditional discrimination tests of uransitive inference by macacjue monkeys: List linking. Joumal of Experimenal Psycho/ogy: Animal Behavior Processes, 22, 105-117.

Weaver, J.E., Steirn, J.N., \& Zentall, T.R. (1997). Transitive inference in pigeons: Control for differential value transfer. Psychonomic Bulletin and Revie'll, 4, 113 117.

Werner, U., Koeppl, U., \& Delius, J.D. (1992). Transitive Inferenz bei nichtverbaler Aufgabendarbietung (Transitive inference with nonverbal task presentation). Zeitschrifi fiir Experimemelle und Angeneandhe Psychologie, 39, 662 093.

Woocher, F.D., Glass, A.L., \& Holyoak, K.J. (1978). Positional discriminability in linear ordcrings. Memory and Cognition, 6, 165-173.

Wynne, C.D.L. (1995). Reinforcement accounts for transitive inference performance. Animal Leaning and Beharior, 23, 207-217.

Wynne, C.D.L. (1997). Pigeon transitive inference: Tests of simple accounts of a complex performance. Behavioural Processes, 39, $95-112$.

Wynne, C.D.L., Fersen, L. von, \& Staddon, J.E.R. (1992). Pigeons' inferences are transitive and the outcome of elementary conditioning principles: A response. Joumal of Experimental Psirchologi: Animal Behavioi Processes, 18, 313-315.

Yuang, F., Cohen, M.A., \& Kincaid, T.G. (1996). Dynamics of a winner-take-all neural network. Neural Nerworks, 9, 1141- 1154.

Zentall, T.R., \& Sherburne, L.M. (1994). Tansfer of value from $S+$ to $S-$ in a simultaneous discrimination. Journal of Experimental Psychology: Animal Behavior Processess, 20, $176-183$.

Zentall, T.R., Sherbume, L.M., Roper, K.L., \& Kraemer, P.J. (1996). Value transfer in a simultaneous discrimination appears to result from within-event Pavlovian conditioning. Journal of Experimenal Psycholog!: Animal Behavior Processes, 22, 68 75. 